\title{
Efficacy of Autologous Platelet Rich Plasma in the Treatment of Male Androgenetic Alopecia
}

\section{Nadia Naeem, ${ }^{1}$ Saadiya Siddiqui, ${ }^{2}$ Atif Shehzad, ${ }^{3}$ Kehkshan Tahir, ${ }^{4}$ Marrium Manzoor}

\begin{abstract}
Objective: To assess the outcome of autologous platelet rich plasma(PRP) for the treatment of androgenetic alopecia in male patients.

Methods: A total of 61 male patients from Department of Dermatology, Lahore General Hospital Lahore with ages between 18-60 years were included in the study during a period of 6 months. PRP was prepared by double spin method and $\mathrm{CaCl} 2$ was used to activate platelets. PRP was injected, with a $30-\mathrm{G}$ needle using Nappage technique. This descriptive case series study was carefully monitored. Protocol included three treatment sessions with an interval of four weeks. Number of hairs, and hair strength was assessed by dermoscopy and hair pull test. SPSS Version-21 was used for data entry and analysis.

Results: The mean age of patients was $42.67 \pm 8.63$ years. Mean number of hair was $21.50 \pm 4.57$ per dermatoscopic field, Post treatment number of hair was $78.10 \pm 16.24$. Mean duration of disease was $10.25 \pm 3.52$ months. Majority of patients were in stage iv $19(31.1 \%)$ and in stage ii $16(26.2 \%)$. There was significant difference in number of hair before and after treatment.

Conclusion: PRP is an effective method to treat the patients with androgen alopecia.

Key Words: Autologus platelet rich plasma, Androgen alopecia.

How to Cite: Naeem N, Siddiqui S, Shehzad A, Tahir K, Manzoor M. Efficacy of autologous platelet rich plasma in the treatment of male androgenetic alopecia. Esculapio.2020;16(04):41-45.
\end{abstract}

DOI: https://doi.org/10.51273/esc20.251649

\section{Introduction}

$\Lambda_{\text {nai }}$ ndrogens have a great effect on scalp and body hair. Scalp hair grows without effect of androgens while body hair growth is dependent on androgen presence. Androgens dependent hair fall is termed as androgenetic alopecia also referred as Male pattern hair loss (MPHL). ${ }^{1}$ Alopecia is a dermatological disorder which is androgen dependent and with genetic predisposition. It generally starts by 20 years of age and effect up to 50 percent of men when they reach by their 50 years of life. ${ }^{2}$ Alopecia involves an ongoing loss and thinning of scalp hair in a specific pattern causing a significant psychological effect on

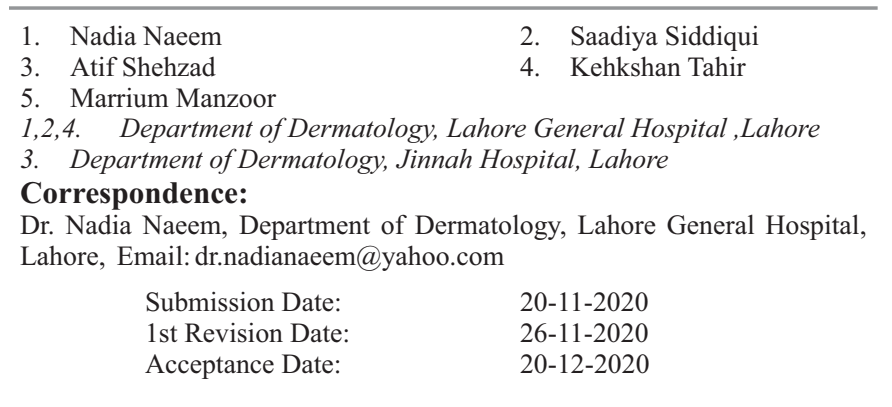

one's mental health and make it difficult for a person to cope up with his self-esteem. The two factors, testosterone metabolite di hydrotesterone and a receptor named as hair follicle related androgen receptor challenging world's dermatologist, as these two are modulating this androgen dependent disorder, whereas few studies have also found genetic involvement in this pathology. ${ }^{3}$ Few treatment option available including minoxidil, 5-alpha-reductase inhibitors and hair transplantation but these modalities have many aftereffect including excessive body hair growth, birth defects, decreased libido and prolonged impotence. ${ }^{4}$ The use of PRP methodology in the skin care and aesthetic medicine may prove as a revolutionary step of dermatological treatment in the near future. However, the available published data is low in count to support this evidence. ${ }^{5}$ Injection of PRP is easy, pocket friendly and convenient mode of method for treating this alopecia which is ensuring great patient overall satisfaction with few documented side-effects. ${ }^{6}$ Rationale of this study is to 
assess the outcome of autologous PRP for the treatment of androgenetic alopecia in male patients in local population. This will improve the way of doing our practice and will also help to update the local management guidelines.

\section{Methods}

This study was conducted at department of dermatology, Lahore general hospital, Lahore. 61 subjects were segregated from outpatient department on the basis of inclusion and exclusion criterion and enrolled in the study after informing them and getting their written consent forms. Name, age and duration of Androgenetic Alopecia of each patient was recorded on properly formulated proforma. Calculation of the sample size was done by considering $95 \%$ confidence level, $12 \%$ margin of error and the estimated percentage of hair pulled out reduction as $65 \%$ on hair pull test ${ }^{7}$. Study Design was Descriptive case series. Duration was 6 months (16-08-2017 to 17-02-2018) and non probability consecutive sampling Technique was used.

Inclusion criteria listed as patients of male gender between ages 18-60 years with mild to moderate androgenetic alopecia with Hmilton-Norwood score 1-5. Exclusion criteria as laid as; any patient not ensuring for follow checkups, on the topical treatment (minoxidil, prostaglandin analogues, retinoids, and corticosteroid) or systemic treatments for Androgenetic Alopecia (such as finasteride, dutasteride, and antiandrogens) since last two months, patients with supressed immunity (malignancy, chemotherapy, steroid therapy), other dermatological disorders involving hair and skin of head, autoimmune disorders, hematologic disorders, platelet dysfunction syndrome and subjects under anticoagulation therapy (on medical record), active infection involving scalp and receiving drugs causing hair loss (retinoids, antidepressants, oral contraceptives, anticoagulants, lipid lowering drugs, B-blockers, ACE inhibitors) were excluded from study. PRP was carefully prepared by method of double spin and $\mathrm{CaCl}_{2}$ poured to mixture in order to stimulate platelets. Affected area of scalp was sterilized with pyodine solution and anaesthetic agent was applied to the specified area which was going to be treated. PRP $\left(0.5-01 \mathrm{ml} / \mathrm{cm}^{2}\right)$ was shifted to a one $\mathrm{ml}$ sterilized syringe with a thirty gauge needle and administered into the selected areas of the scalp using Nappage technique in a depth of 1.5-2.5mm of skin. Our protocol included 3 treatment sittings after every 4 weeks. At each visit the number of hair monitored by dermatoscope (3Gen DermLite) using maximum magnification(10x) as standard and by gross photographs. Hair number in one dermatoscopic field using maximum magnification was counted at each visit. Strength of hair was assessed using hair pull test with more than $10 \%$ of hairs removed in a plucked bunch of hair considered positive. SPSS Version-21 was used to analyse data. The quantitative variables i.e. age, number of hair and duration of androgenetic alopecia was presented as Mean and Standard deviation. Data was stratified for age, duration of Androgenetic Alopecia and stage of alopecia to address the effect modifiers. In order to check the study significance, post stratification Independent tTest was applied taking P-value $\leq 0.05$ as significant.

\section{Results}

The mean age of patients was $42.67 \pm 8.63$ years. Mean number of hair before treatment was $21.50 \pm$ 4.57 per dermatoscopic field at maximum magnification and it was $78.10 \pm 16.24$ post treatment (P-value $<0.05$ ) (Table 1). Mean duration of disease was 10.01 \pm 3.46 months. Majority of patients were in stage ii, iii, and stage iv i.e. $16(26.2 \%) 15(24.6 \%)$ and $19(31.1$ $\%)$ respectively. In stage $i$ and $v$ there were $1(1.6 \%)$ and $10(16.4 \%)$ patients respectively(Graph 1). Post treatment Hair pull test was negative in majority of the cases $48(78.7 \%)$ and mean hair pull score was significantly different pre (90.57) and post treatment (78.45) with $\mathrm{p}$-value $<0.05$ (Table 1 ). When data was stratified for the age it was noted that $19(76 \%)$ patients were in the age groups of 18-40 years and $29(80.6 \%)$ in $41-60$ years age groups was having negative hair pull test after treatment. There was no significant impact of the duration of the disease for the negative hair pull test and stages of androgen alopecia (Table 2). Figure\#1 shows pre and post treatment dermatoscopic and gross photographs of few patients.

Table 1: Descriptive statistics of Mean number of hair per dermatoscopic field(DF), Hair Pull Test status and Mean Hair Pull Score

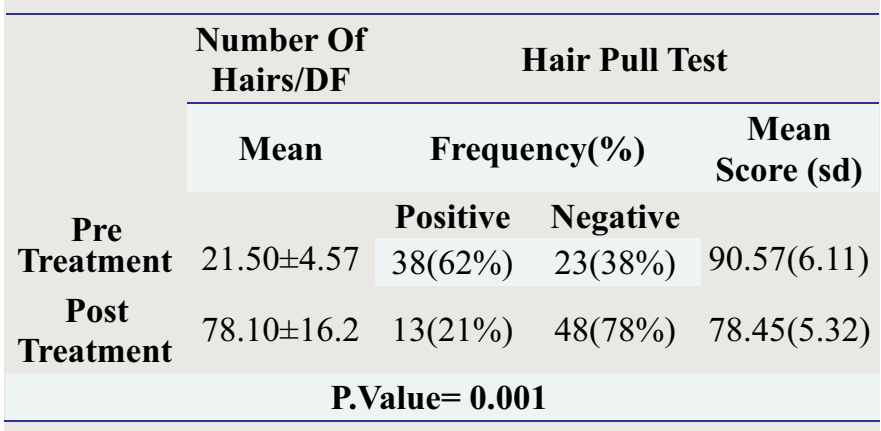


Table 2: Stratification of the Hair Pull Test with Respect to different Baselines Parameters

\begin{tabular}{lcccc}
\hline & & \multicolumn{2}{c}{ Hair Pull Test } & P- \\
\cline { 3 - 4 } Age & Positive & Negative & value \\
& $18-40$ year & $6(24 \%)$ & $19(76 \%)$ & 0.75 \\
Duration of & $41-60$ year & $7(19.4 \%)$ & $29(80.6 \%)$ & \\
Disease & $1-6$ Month & $1(8.3 \%)$ & $11(91.7 \%)$ & 0.43 \\
Group of Androgen & $>6$ month & $12(24.5 \%)$ & $37(75.5 \%)$ & \\
Alopecia & $>$ iii & $7(21.9 \%)$ & $25(78.1 \%)$ & 0.98 \\
\hline
\end{tabular}

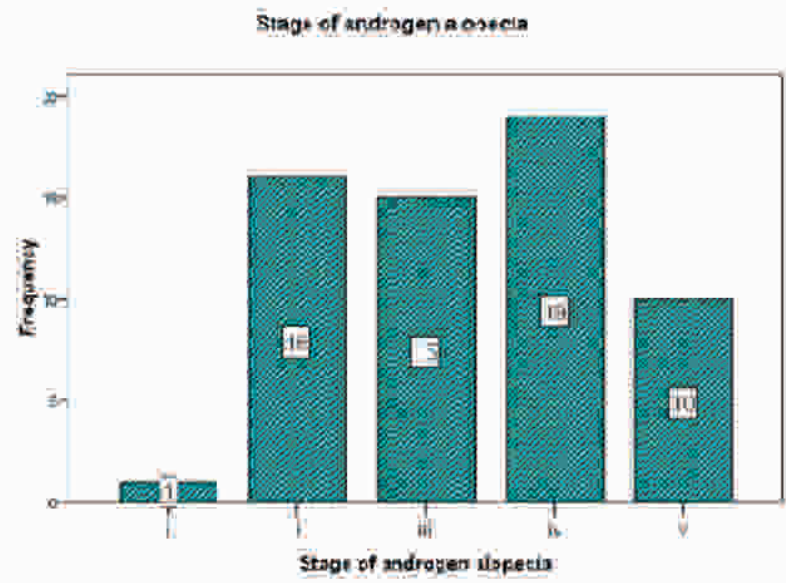

Graph 1: Presentation of the Stages of the Androgen Alopecia in the Study Subjects

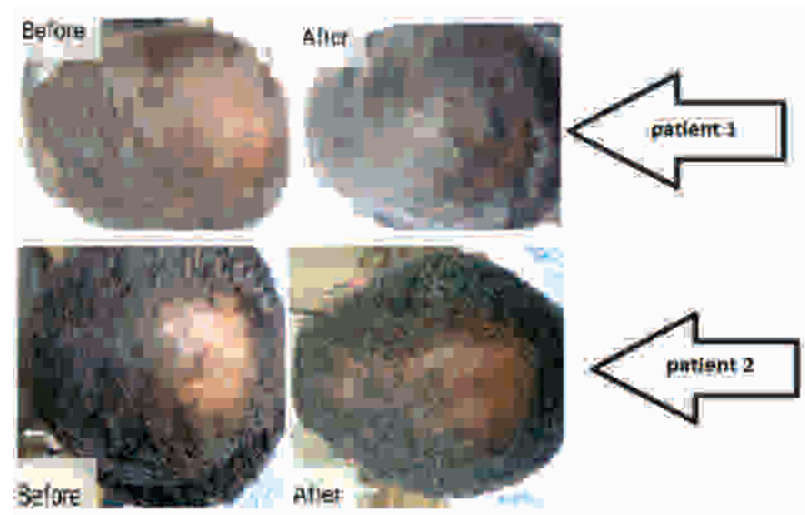

Figure 1: Dermatoscopic and Gross photoghraphs of study population

\section{Discussion}

Platelet-rich plasma (PRP) is considered as a novelty in the field of biotechnology. It is a combination of cell-based therapy and tissue engineering. This therapy outlines an autologous preparation of plasma with distilled platelets. This mixture of platelet has a magical power to regenerate and repair the damaged body sites as it contain numerous factors of growth and cytokines. ${ }^{8}$ Many medical specialities are getting benefits from this new technique including

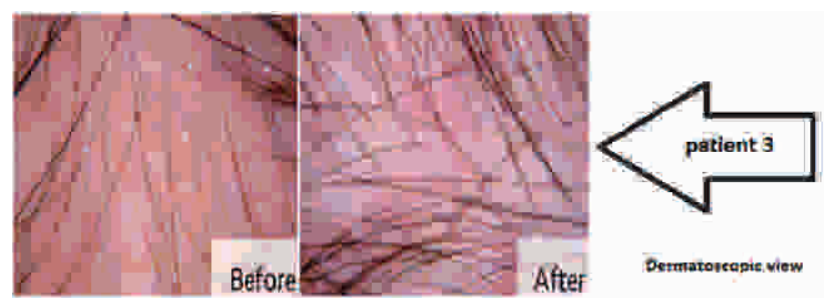

periodontal therapy, maxillofacial surgery, orthopedics and sports medicine. For few recent years, dermatologist are laying their hands on this new modularity, particularly in relieving acne scars, fat grafting, wound healing and hair regrowth.' Researches has explored many beneficial outcomes of PRP in adipose precursor cell proliferation, wound healing, differentiation of cell and forming new blood vessels. A normal blood sample contains a proportion of around $93 \%$ red blood cells, $6 \%$ platelets and $1 \%$ white blood cells. However, what scientist do while preparing PRP, the reverse this proportion i.e. a $94 \%$ concentration of platelets and a 5\% concentration of RBCs by centrifugation and it's the principal for making this incredible mixture. The exceptional high concentration of useful growth factors and cytokines in PRP basically make this mixture the most suitable selection for facilitating tissue rejuvenation and healing. The concentration of platelets with tissue reparative efficacy is found to be nearly 1 million platelets $/ \mu 1$, which is 5 times the normal concentration of platelets. ${ }^{10}$ Losing a body part, of any proportion, does have a negative impact on one's mind but especially losing hair means losing a chunk of your integrated personality and it does have a great impact on one's psychological health. Unfortunate is that, this androgen dependent hair loss have very few treatment option including topical minoxidil and oral fenasteride (appoved by FDA) having many side effects, ${ }^{11}$ making it more challenging for dermatologists to treat it. PRP has already succeeded in drawing attention of many physicians and surgeons all around the world such as in plastic surgery, orthopaedic surgery and cardiac surgery because of its potential use in skin rejuvenating effects, rapid healing, reduced infection rate and decreased chance of hypertrophic keloids and scars. ${ }^{12,13}$

Certain growth factors are found to activate the proliferative phase and trans-differentiation of hair and stem cells and produce new follicular units. Papilla cells get proliferated under the influence of basic fibroblast growth factor (bFGF) in vitro and thereby plays a key role in elongating hair shaft. ${ }^{14}$ Our 
research work has reported that after performing three sessions of PRP the hair pull test becomes negative. This outcome is similar to the outcome of a study done by Bestiet al. ${ }^{15}$ Who also have observed improvement in hair volume and coverage but according to our study, only moderate improvement in hair volume and coverage was observed by using dermatoscope to monitor it. In the previous studies, One of the studies show that baseline mean hair count $89.6 \pm 20.9$ and at 3 months mean hair count was $123.2 \pm 33.7 .{ }^{16}$ We have a little variation in the results of this study as compared to the previous study due to generalizability of the study population. A specific population which was studied previously had a pattern of alopecia while in our study it was advantage that we have to deal with the general population. Another study done by V.cervellietal showed baseline mean hair count $103.6 \pm 30.7$ and at end of 3 months a mean increase was $121.6 \pm 34.1{ }^{7}$ One study showed $100 \%$ positive hair pull test before treatment and pull test was negative in $81.81 \%$ at end of treatment and There is a marked increase of usual number of 71 hair follicles to 93 hair follicle units. ${ }^{2,6}$ As sample size is an important factor for the validity of the results so the sample size in this study was high as compared to the previous studies. ${ }^{6,716}$ Previously most of the studies were conducted on a sample size of less than 30 cases but in our study the sample size was 61 cases that is the advantage of this study. These findings of our are further supported by the results of a case-control study which states that there's a notable increase in hair number per $\mathrm{cm}^{2}$ after treating subjects with PRP injection as compared to their controls (mean difference [MD] 14.38, 95\% confidence interval $[\mathrm{CI}] 6.38-22.38, \mathrm{P}<0.001)$. Likewise, a crucial growth of hair thickness cross section per $10-4 \mathrm{~mm}^{2}$ (MD 0.22, 95\% CI 0.07-0.38, P=0.005) added another plus point to PRP treated group. However study concluded that there isn't significant positive effect of PRP injection on under-study subjects, in terms of getting benefits in increase hair number (MD 18.79\%, 95\% CI - 8.50-46.08, P = 0.18 ), neither with hair thickness (MD 32.63\%, 95\% $\mathrm{CI}-16.23-81.48, \mathrm{P}=0.19)$ which favours the results of current study. ${ }^{17}$ In previous study lesser time was given and a booster dose was administered at the last injection which has led to biasness and changed results. But in our study almost same concentration of the plasma is administered so that actual results could be obtained. There's a published study who reported that their subjects have shown good results in term of increased hair density at six weeks $(154.80 \pm 34.39$ hairs per $\left.\mathrm{cm}^{2}\right)$, at three months $(170.70 \pm 37.81$ hairs per $\left.\mathrm{cm}^{2}\right)$ and at six months $(156.23 \pm 37.75$ hairs per $\mathrm{cm}^{2}$ ), we did not have so long follow up, but the results were similar. ${ }^{18}$ As it was a meta-analysis so it further strengthens the results of our study. Hence, on the basis of this study the PRP treatment could be a method of the choice in androgenic alopecia.

At present, we scientist have very limited published data regarding PRP's probable outcomes on hair growth. This study has added to that limited data. But still it should be conducted on a larger scale with involvement of the multiple hospitals/institutions. Moreover, in this study effects of the environment and hygienic conditions are not evaluated so further studies are needed.

\section{Conclusion}

Our study hereby concludes that treating the patients of androgenic alopecia with PRP injectable comes out to be an effortless, pocket-friendly and practicable treatment modality for hair loss and it appear to be a novel valuable adjuvant treatment option for alopecia. With adequate published data as evidence for its effectiveness, this treatment option is still rooting itself in medical sciences. Clinical trials are fewer but due to its easy going approach and availability, it's an outstanding treatment option for worldwide dermatologists.

\section{Conflict of Interest: $\quad$ None}

\section{References}

1. Kaufman KD. Androgens and alopecia. Mol Cell Endocrinol. 2002 Des 30;198(1-2):89-95.

2. Singhal P, Agarwal S, Dhot PS, Sayal SK. Efficacy of platelet-rich plasma in treatment of androgenic alopecia. Asian J Transfus Sci. 2015 Jul-Dec;9(2): 15962.

3. Rallis E, Falidas E, Villias C. Amyopathic dermatomyositis-associated bilateral elbow ulcers successfully treated with autologous platelet-rich plasma. Int J Dermatol. 2014 Jan;53(1):e50-e2.

4. Kang BK, Lee JH, Shin MK, Kim NI. Infraorbital rejuvenation using PRP (platelet-rich plasma): A prospective, randomized, split-face trial. J Am AcadDermatol 2013;68:SAB24.

5. Arshdeep, Kumaran MS. Platelet-rich plasma in 
dermatology: Boon or a bane? Indian J Dermatol VenereolLeprol. 2014 Jan-Feb;80(1):5-14.

6. Khatu SS, More YE, Gokhale NR, Chavhan DC, Bendsure N. Platelet-rich plasma in androgenic alope-cia: myth or an effective tool. J CutanAesthetSurg . 2014 Apr;7(2):107-10.

7. Cervelli V, Garcovich S, Bielli A, Cervelli G, Curcio $\mathrm{B}$, Scioli M, et al. The effect of autologous activated platelet rich plasma (AA-PRP) injection on pattern hair loss: clinical and histomorphometric evaluation. Biomed Res Int. 2014;2014.

8. Marwah M, Godse K, Patil S, Nadkarni N: Is there sufficient research data to use platelet-rich plasma in dermatology? Int J Trichology 2014;6:35-36.

9. Dhillon RS, Schwarz EM, Maloney MD: Plateletrich plasma therapy - future or trend? Arthritis Res Ther 2012;14:219.

10. Oh DS, Cheon YW, Jeon YR, Lew DH: Activated platelet-rich plasma improves fat graft survival in nude mice: a pilot study. DermatolSurg 2011;37:619625 .

11. Olsen EA, Weiner MS, Delong ER, Pinnell SR. Topical minoxidil in male pattern baldness. J Am AcadDermatol. 1985;13:185-92.

12. Gardner MJ, Demetrakopolous D, Klepchick PR, Mooar PA. The efficacy of autologous platelet gel in pain control and blood loss in total knee arthroplasty. An analysis of the haemoglobin, narcotic requirement and range of motion. IntOrthop. 2007; 31:30913.

13. Glover JL, Weingarten MS, Buchbinder DS, Poucher RL, Deitrick GA, 3rd, Fylling CP. A 4-year outcome based retrospective study of wound healing and limb salvage in patients with chronic wounds. Adv Wound Care. 1997; 10:33-8.

14. Katsuoka K, Schell H, Wessel B, Hornstein OP. Effects of epidermal growth factor, fibroblast growth factor, minoxidil and hydrocortisone on growth kinetics in human hair bulb papilla cells and root sheath fibroblasts cultured in vitro. Arch Dermatol Res. 1987; 279:247-50.

15. Besti EE, Germain E, Kalbermatten DF, Tremp M, Emmenegger V. Platelet-rich plasma injection is effective and safe for the treatment of alopecia. Eur J Plast Surg. 2013;36:407-12

16. Gentile P, Garcovich S, Bielli A, Scioli MG, Orlandi A, Cervelli V. The effect of platelet rich plasma in hair regrowth: A randomized placebo controlled trial. Stem Cells Transl Med. 2015 Nov;4(11):1317-1323

17. Giordano S, Romeo M, Di Summa P. A meta-analysis on evidence of platelet-rich plasma for androgenetic alopecia. International journal of trichology. 2018 Jan; 10(1):1.

18. GkiniMA,KouskoukisAE,Tripsianis G etal. Study of platelet-rich plasma injections in the treatment of androgenetic alopecia through an one-year period. J CutanAesthetSurg 2014;7:213-219.

\section{Authors Contribution}

NN: Research, Statistics, compliation of paper, practical work

SS: Supervisor, compliation of paper

SA: Supervisor, arrangement of all gadgets, selection of topic

TK: Supervisory Role 